\title{
Collaboration Networks Involving Humanitarian Organisations - Particular Problems for a Particular Sector
}

\author{
Aurélie Charles ${ }^{1}$, Matthieu Lauras ${ }^{1,2}$, and Rolando Tomasini ${ }^{3}$ \\ ${ }^{1}$ Université de Toulouse - Mine Albi, Campus Jarlard, Route de Teillet, \\ 81013 Albi CT Cedex 09, France \\ ${ }^{2}$ Toulouse Business School, 20, Bd Lascrosses - BP 7010 - 31068 Toulouse Cedex 7, France \\ ${ }^{3}$ INSEAD, Bd de Constance, 77300 Fontainebleau, France \\ \{aurelie.charles, matthieu. lauras\} @mines-albi.fr, \\ rolando.tomasini@insead. edu
}

\begin{abstract}
There is no relief operation similar to another. Nature, number and incentives of stakeholders vary tremendously from one operation to another, and so do the collaboration modes. One thing is always true thought: for each disaster, collaboration networks are implemented on both global and local levels. Yet, lack of collaboration is often underlined as a major weakness of humanitarians. Practitioners have therefore recently focused on this issue in order to find improvement paths. This paper, using a review of existing academic literature as well as reports published by practitioners, illustrates how collaborative networks are designed in the humanitarian sector. A panorama of existing collaboration networks during and in-between relief operations is presented. Barriers and enablers to implement them are made explicit. Two frameworks are finally proposed to better understand collaboration modes at a local level on one hand, and collaboration protocols at a global level on the other hand.
\end{abstract}

Keywords: Humanitarian, Disaster Relief, Collaborative Networks, Collaboration, Coordination.

\section{Introduction}

Every year, disasters are impacting more than 210 million people, and year after year, their frequency increases. So is the number of Non Governmental Organizations (NGOs) created to help those in need before or after a disaster. Ten years ago, most of the time, humanitarian actors in the field had a really limited knowledge of what others were doing. This kind of knowledge is still very difficult to gather and spread, especially because there are so many stakeholders involved. Yet, many improvements have been made recently, driven both by field necessities and by humanitarian organizations' professionalization. This article aims at illustrating and analysing the collaboration networks that are implemented in the context of humanitarian aid. As humanitarian organisation work as well between disasters than on the field, during relief operations, we will also illustrate and analyse the collaboration networks that are build on a global, long term perspective. This picture of the actual situation is a first, but needed step 
before any proposal of improvement. It complements the recent research applications in the humanitarian sector, which usually give priority to more technical approaches, without much consideration of their applicability. [1] Various factors, such as local capacity or nature and number of stakeholders involved are influencing the design of collaboration networks in such a difficult context. Concretely, the paper proposes an overview of practices in terms of collaboration networks in a context of relief operations, both at local and global levels. For each level, a framework is developed in order to classify collaboration modes, means and applicability.

\section{Collaboration Networks on a Local Level in the Context of Relief Operations}

\subsection{Overview of Existing Modes}

On a local level, we call collaboration network "the system-wide structure of interorganisational coordination during humanitarian operations."[2] Donini [3] has identified three categories of collaboration in the context of relief operations:

- Coordination by command where there is central coordination; agreement on responsibilities and objectives; and common territorial areas of responsibility.

- Coordination by consensus where organizations have access to compatible or shared communications equipment, liaison and interagency meetings and premission assessments.

- Coordination by default includes routine contact between desk officers and civil military operations centers.

We will use those definitions, but detail the conditions under which they can be implemented or not. Figure 1 shows a simplified picture of usual collaboration modes. Many stakeholders with various incentives are involved in operations (see figure 2). And the bigger the disaster, the bigger the number of NGOs that will have sufficient funding to participate to the humanitarian response... and the more difficult coordination will be. The 2004 Indian Ocean Tsunami is one example of the chaos that can happen when auto-regulation does not take place. Many factors, from the magnitude of the disaster to the presence of many tourists "generated massive media attention which in turn prompted an inordinate public response to donate money as people felt a moral obligation to help. There was also an unprecedented wave of governments' attention which was not necessarily free of a political agenda." [4]

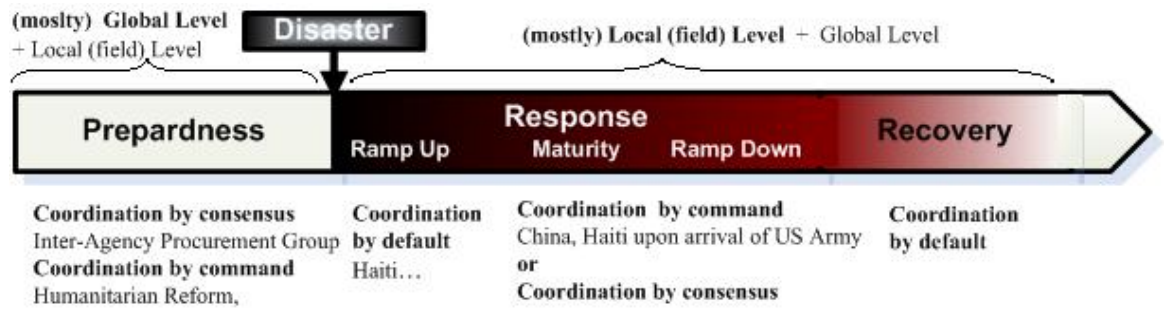

Fig. 1. Disaster phases and collaboration modes (Inspired by [5] and [3]) 


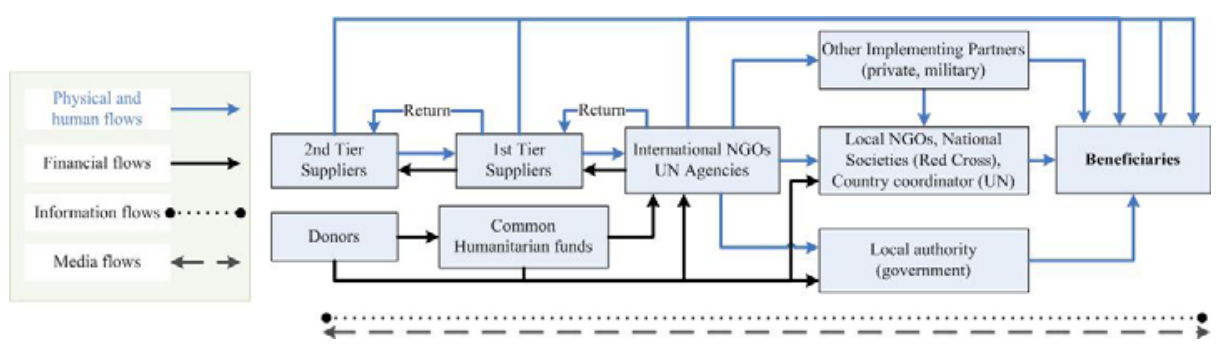

Fig. 2. Stakeholders involved in global relief efforts

"Due to the lack of adequate regulation and the presence of too many players, chaos ensued in Sumatra after the tsunami." [4] The presence of a local authority able and willing to act as central coordinator is a first element that shapes the relief operations. The presence of the army, local or not, also impacts the collaboration networks. They often act as central coordinator and therefore impose a coordination by command (see figure 3). Pettit and Beresford [6] have detailed the relations between military and humanitarian organizations. UN agencies also sometimes act as such. See [7]; [8] and [9] for details of centralized coordination around a UN Agency. Recent publications have applied "organizational design" best practices to humanitarian relief, thought they focus on a specific country and study only the response phase, like [10] and [11].

Thus, the diversity of stakeholders and the variability of their presence and strength from one disaster to another makes it really difficult sometimes to find and implement the appropriate collaboration network. Even usual humanitarian organisations, though they share the same humanitarian principles, may be reluctant to partner each other. The neutrality and impartiality imposed by the humanitarian space has already made MSF (among others) refuse to work with some governments, and sometimes also UN agencies. It is therefore difficult, if not impossible for NGOs to have formal coordination processes with hierarchical links that would make them report to governmental agencies or other humanitarian stakeholders. Similar issues can also be faced between actors, that would not appear much different at first glance. The French Red Cross and IFRC, for example, share the same name, but that doesn't mean that they accept clear reporting lines.

Fortunately, if we consider only humanitarian organizations offering the same range of products and services and having a shared initial intent, collaboration is more frequent. They realize that "in a world of scarce resources, although humanitarian action has no price, it obviously has a cost, and an improved management of this cost has an influence on the ability to send relief to a varying number of operation sites. Among possible savings, the best logistical coordination plays a significant part, for example in trying to avoid useless equipment or food redundancies in one place when a few miles further, both are sorely lacking.'[12] Such successful collaboration networks on local level often include local partners. Because they know the local customs and networks, because they are really useful to capture knowledge of the local environment, locals are in a position to complement international staff. Those implementing partners can be local NGOs or local companies with an existing network and trained resources. During the 2006 Lebanon conflict, Agility, a logistics company, has been a major stakeholder. "We had decided 
that we were going to provide in-kind services in the form of transportation and warehousing, and also offer the expertise of some experienced operational managers to humanitarian organisations, since we knew that the logistics landscape of Syria and Lebanon would be unfamiliar to many humanitarian actors." [13] In such environments, local knowledge is essential. "The minute something happens, you see, you listen... you are used to working with difficult, uncertain and constantly changing conditions" [13] With a turnover of $80 \%$ per year, international NGOs have limited trained resources. Local implementing partners are therefore often really useful. Yet, their presence and strength vary from one disaster to another. Collaboration networks vary accordingly. The Table 1 summarizes the main barriers and enablers to implement collaboration networks involving humanitarian organizations on a local level.

Table 1. Barriers and enablers to implement collaboration networks involving humanitarian organizations on a local level, inspired from [14] [5] [15] and [16]

\begin{tabular}{ll}
\hline \multicolumn{1}{c}{ Barriers } & \multicolumn{1}{c}{ Enablers } \\
\hline $\begin{array}{l}\text { In-country NGOs vary widely in their ability and } \\
\text { willingness to partner UN or International NGO } \\
\text { bodies }\end{array}$ & $\begin{array}{l}\text { Most organisations are connected to } \\
\text { one another in principle through their } \\
\text { desire to provide aid effectively }\end{array}$ \\
\hline $\begin{array}{l}\text { Most organisations are tied to each other only } \\
\text { episodically }\end{array}$ & $\begin{array}{l}\text { General awareness of the aims and } \\
\text { competencies of principals actors }\end{array}$ \\
\hline $\begin{array}{l}\text { Accurate data, for need assessment, logistics } \\
\text { management and many other critical part of } \\
\text { operations is vital but typically difficult to obtain }\end{array}$ & $\begin{array}{l}\text { Specific shared IT tools are developed } \\
\text { to improve data capture and analysis }\end{array}$ \\
\hline $\begin{array}{l}\text { All humanitarian organisations are poor in lessons } \\
\text { learnt and need structure to prepare knowhow, } \\
\text { knowledge rules/pools, to clarify what they need in } \\
\text { specific fields }\end{array}$ & $\begin{array}{l}\text { Score Cards are under development in } \\
\text { most major International NGOs }\end{array}$ \\
\hline $\begin{array}{l}\text { The humanitarian community has many serious } \\
\text { weaknesses in managing human resources, from } \\
\text { recruitment to training to appraisal }\end{array}$ & \\
\hline
\end{tabular}

\subsection{Proposition to Support the Collaboration on a Local Level}

"Each State has the responsibility first and foremost to take care of the victims of natural disasters and other emergencies occurring on its territory. Hence, the affected State has the primary role in the initiation, organization, coordination, and implementation of humanitarian assistance within its territory." [17] In the field, after a disaster, collaboration networks involving humanitarian organisations have to include local governments. It is their ability and willingness to take the lead or not, that shape the global humanitarian relief effort. [14] [17] Then, the strength of the local capacity comes into consideration. "If trained resources and adequate means of actions are already ready to be deployed, they should be and usually are involved from the beginning."[14] Local collaboration networks involving humanitarian organizations can therefore take many forms, depending on the nature, number and incentives of stakeholders involved. The selection of the most appropriate collaboration mode is never easy, particularly in a context of humanitarian crisis. We have drawn up a brief panorama of various existing collaboration modes and their 
applicability on a local level, stressing the diverse barriers and enablers to set them up. Based on those elements, we propose a framework that explains the different steps which enable, in a given situation, to choose the appropriate collaborative mode on local level (see Figure 3).

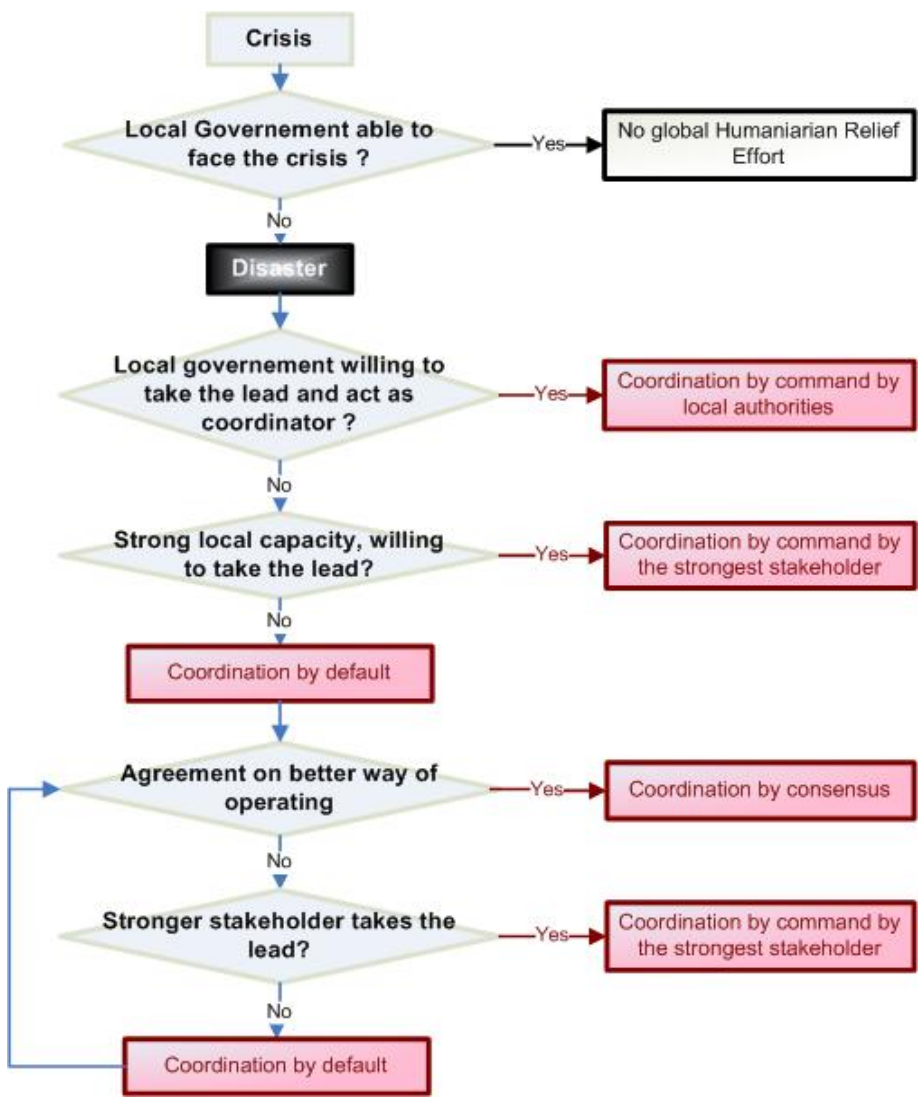

Fig. 3. Choice of local level collaboration modes during the response phase

\section{Collaboration Networks on a Global Level}

\subsection{Overview of Existing Modes}

More and more stand alone initiatives are giving birth to global collaboration networks. The Global Humanitarian Platform, for example, has been created in 2006 to strengthen partnership between humanitarian actors. The red cross and red crescent movement, NGOs and UN agencies are thus meeting once a year to increase their transparency and complementarities. We can also add more dedicated group meetings, like the Inter-Agency Procurement Group or the Fleet Forum. Those gather practitioners to share best practices and align on key improvement areas, like 
procurement or fleet management. There again, academics working on those specific subjects could be much helpful. Indeed, some partnership with private companies are already under development. Many case studies illustrated such successful approaches. See [13][18][19] and [5] among others. More recently, partnerships have begun to include other humanitarian actors, such as governmental agencies like the Centre National d'Etudes Spatiales (CNES) and also academic partners. One of those publicprivate partnership is Huma-Nav. It aims at developing a dedicated service for humanitarian fleet management, enabling to share information on mutual experiences and existing initiatives[20]. Those are a few chosen examples of collaboration networks involving humanitarian organizations. Many others exist, including other actors like donors or suppliers. "Such partnerships are interesting, challenging and rewarding... but really difficult sometimes. It is essential to choose the right partners and find the optimal number of actors"[14]. Table 2 summarizes the major barriers and enablers to implement such collaboration networks.

Table 2. Barriers and enablers to implement collaboration networks involving humanitarian organizations on a global level, inspired from [5] [21] and [20]

\begin{tabular}{|c|c|}
\hline Barriers & Enablers \\
\hline $\begin{array}{l}\text { Lack of mutual understanding due the } \\
\text { diversity of actors }\end{array}$ & Choice of the right ecosystem of actors \\
\hline Lack of transparency and accountability & $\begin{array}{l}\text { Incentives for shared information on mutual } \\
\text { experiences and existing initiatives }\end{array}$ \\
\hline Insufficient commitment on all levels & Involvement of key actors of the value chain \\
\hline Lack of clarity on roles and responsibilities & $\begin{array}{l}\text { Develop clear and jointly agreed roles and } \\
\text { responsibilities to encourage commitment of } \\
\text { actors }\end{array}$ \\
\hline Lack of change management & Participatory approach \\
\hline $\begin{array}{l}\text { Lack of funding for activities that have no } \\
\text { direct, visible and dedicated field application }\end{array}$ & $\begin{array}{l}\text { Support of adequate Information Management } \\
\text { tools and services }\end{array}$ \\
\hline
\end{tabular}

\subsection{Particular Case: The Humanitarian Reform}

In 2005, The United Nations Emergency Relief Coordinator, Jan Egeland asked four independent consultants to identify the factors that have hindered the speed and effectiveness of humanitarian response in the past and to propose appropriate steps to improve the timeliness and impact of future humanitarian interventions. [22] This has lead to the 2005 Humanitarian Reform. Its aims are as follow:

- $\quad$ Strengthening of the response capacity: the Cluster Approach

The response is organized per sector or area of activity (Camp Coordination, Logistics, but also Education, etc.) Each sector has its cluster leads well identified on a global level. Then, for each emergency, different local clusters leads are chosen.

- $\quad$ Better Humanitarian Financing: the Central Emergency Response Fund (CERF). It is a cash-flow mechanism, loaned to enable quick access to funds and a stand-by fund, granted for rapid response and under-funded emergencies (response only). 
- $\quad$ Strengthening of the Humanitarian Coordination System

The aim is the creation of a poll of human resources equipped with the right skills and experience and the development of a Score-Card. It is still under development.

Most of the UN community agrees that its implementation constitutes an improvement, but many others consider that there are many challenges remaining. The competitive funding environment, focused on the response phase instead of longterm, more sustainable improvements remains a major issue [23]. The use of local NGOs and cluster partners is also far from systematic [24] and therefore remains a challenge. Change management is never easy, and in this case, many NGOs, local or international felt disregarded or decided to by-pass this new organization proposed by the United Nations. And those who accepted this reform still lack a rationalized coordination structure. "Clusters were hampered by a lack of full attendance at meetings and problems with, for example, operational/field staff located at hub clusters while decision-makers, such as heads of agencies in Islamabad, a problem compounded by communication problems between hubs and clusters."[25] Best practices exist, but the management of this knowledge is still dependant on the presence and training of local capacity. "In Union of Myanmar, a dedicated Information Manager has been appointed and a web based information management system has been developed, enabling agencies to search and access relevant information for the response, including digitalized maps showing operational agencies by geographical area."[21] Yet, many reports for other emergencies pinpoint the "need for adequate IM tools and services to support the approach."[24] Those are areas that research on collaboration networks could focus on.

\subsection{Proposition to Support the Collaboration on a Global Level}

The two previous parts clearly illustrated the difficulty to have a pre-defined response model to implement no matter where and what the disaster is. To become more effective and more efficient in their response, humanitarians seem to want to develop a kind of collaborative protocol at a global level (see the UN experience). Our purpose is to propose a framework that should be considered as a first step to support the different stakeholders in designing such a protocol.

First, as we have described before, humanitarian organizations have to control globally their operations. But because they are under-resourced, they have to define priorities in order to dispatch properly funds and, of course, human resources. This is a problem of balancing of mobilization and affectation activities. Secondly, because a humanitarian supply chain is made up of several partners (private companies, army, humanitarians...), the coordinator (see part 2.) has to guarantee the coherence and efficiency of the network. Each stakeholder must synchronize its actions in order to be more effective and reactive. Globally, this is a problem of synchronization. The last thing that appears in the previous literature review is that the two precedent components do not relate to the preparedness phase of the lifecycle (see Figure 1). Actually, during the preparedness phase, all humanitarian stakeholders should capitalize and share on their past experiences in order to define best practices in terms of supplier selections, business processes, skill management, etc. Collaboration at a global level could enable a cross-learning between stakeholders and ensure the use of best practices during future operations. Globally, this is a problem of training. 
To summarize, the design of a collaborative protocol at a global level seems to include three major components:

1. Balancing: mobilize and affect properly funds and skills for on-going crises;

2. Synchronization: guarantee coherence and efficiency on a relief operation;

3. Training: facilitate cross-learning between network members and implementation of best practices.

\section{Conclusion and Future Works}

This paper has detailed various collaborations modes and their applicability in the context of disaster management. It has illustrated the particularities of the humanitarian sector and the specific problems they create. Our approach is a first step aiming at a better understanding of those particularities by proposing two frameworks for supporting collaboration at a local level and global level. It sets the basis for successful applications of research works on collaboration network in this sector.

\section{References}

1. Schmenner, R.W., Wassenhove, L.V., Ketokivi, M., Heyl, J., Lusch, R.F.: Too much theory, not enough understanding. J. Oper. Manage. 27, 339-343 (2009)

2. Moore, S., Eng, E., Daniel, M.: Spencer, International NGOs and the Role of Network Centrality in Humanitarian Aid Operations: A Case Study of Coordination During the 2000 Mozambique Floods. Disasters 27, 305-318 (2003)

3. Donini, A.: The policies of mercy: UN coordination in Afghanistan, Mozambique and Rwanda (1996)

4. Tomasini, R., Van Wassenhove, L.N.: Humanitarian Logistics. PalgraveMacmillan, Oxford (2009)

5. Van Wassenhove, L.N.: Humanitarian aid logistics: supply chain management in high gear. Journal of the Operational Research Society 57, 475-489 (2006)

6. Pettit, S.J., Beresford, A.K.C.: Emergency relief logistics: an evaluation of military, nonmilitary and composite response models. Int. J. of Logistics: Res. App. 8, 313-331 (2005)

7. Kent, R.C.: The United Nations' Humanitarian Pillar: Refocusing the UN's Disaster and Emergency Roles and Responsibilities. Disasters 28, 216-233 (2004)

8. De Mul, E.: Coordination of humanitarian aid-a UN perspective. The Lancet 360 (2002)

9. Van Wassenhove, L.N., Samii, R.: The United Nations Joint Logistics Centre (UNJLC): the genesis of a humanitarian relief coordination platform. INSEAD Case Study (2003)

10. Benini, A.A.: Network Without Centre? A Case Study of an Organizational Network Responding to an Earthquake. Journal of Contingencies Crisis Management 7, 38 (1999)

11. Stephenson, M.: Making humanitarian relief networks more effective: operational coordination, trust and sense making. Disasters 29, 337-350 (2005)

12. Chandes, J., Paché, G.: Investigating humanitarian logistics issues: from operations management to strategic action. J. of Manuf. Technol. Manage. 21, 320-340 (2010)

13. Tomasini, R.M., Hanson, M., Van Wassenhove, L.N.: Agility: A Global Logistics Company and Local Humanitarian Partner. INSEAD Case Study (2008)

14. Humanitarian Workers Interviews (2010)

15. Stephenson, M.: Toward a Descriptive Model of Humanitarian Assistance Coordination. Voluntas: Int. J. of Voluntary and Nonprofit Organizations 17, 40-56 (2006) 
16. ICVA The Evolving UN Cluster Approach in the Aftermath of the Pakistan Earthquake: An NGO Perpective, http: / / www . icva.ch/doc00001756.html

17. United Nations, Strengthening of the coordination of humanitarian emergency assistance of the United Nations, General Assembly Resolution 46/182. In: 78th plenary meeting (1991)

18. Tomasini, R.M., Van Wassenhove, L.N.: Logistics firms and relief agencies: learning from each other. INSEAD Quaterly 14 (2005)

19. Van Wassenhove, L.N.: Fleet forum: rethinking humanitarian vehicle management (2006)

20. Faucher, P.: Space-based applications for development, disaster management, emergency response, humanitarian action. In: Third Int. UN-SPIDER Workshop, Bonn (2009)

21. Boughen, L., LeTurque, H.: Implementation of the WASH Cluster Approach (2008)

22. UNOCHA: Humanitarian Response Review (2005), http: / / www . reliefweb. int

23. Jahre, M., Heigh, I.: Does failure to fund preparedness mean donors must prepare to fund failure in humanitarian supply chains? NOFOMA, Helsinki (2008)

24. UNOCHA: Building a Stronger, More Predictable Humanitarian Response System (2005)

25. ActionAid International: The Evolving UN Cluster Approach in the Aftermath of the Pakistan Earthquake: An NGO perpective (2006),

http: / /www. icva.ch/doc00001756.html 